www.nature.com/pj

\title{
Synthesis and study of polycyanurates based on 2-carbazol-4,6-dichloro-s-triazine
}

\author{
Shahrukh T Asundaria, Vinodchandra B Patel and Keshav C Patel
}

Eight polycyanurates have been synthesized by the interfacial polycondensation of 2-carbazol-4,6-dichloro-s-triazine with 1,7-dihydroxynaphthalene (DHN-1,7), 1,4-dihydroxyanthraquinone (DHA-1,4), ethylene glycol (EG), diethylene glycol (DEG), triethylene glycol (TEG), 1,2-propanediol (Pr-1,2), 1,4-butanediol (Bu-1,4) and 1,8-dihydroxyanthraquinone (DHA-1,8). All of the polycyanurates thus synthesized were characterized for their solubility, density, viscosity, infrared spectra, nuclear magnetic resonance spectra and thermogravimetric analysis. Chlorinated solvents were found to be good solvents for all of the polymers. The density of the polycyanurates varied from 1.186 to $1.247 \mathrm{gm} \mathrm{cm}^{-3}$. The intrinsic viscosity of the polycyanurates followed the sequence given below: homopolycyanurates of DHN-1,7 (HPDHN-1,7) > homopolycyanurates of DHA-1,8 (HPDHA1,8) > homopolycyanurates of DHA-1,4 (HPDHA-1,4) > homopolycyanurates of EG (HPEG) $>$ homopolycyanurates of DEG (HPDEG) $>$ homopolycyanurates of TEG (HPTEG) $>$ homopolycyanurates of Bu-1,4 (HPBu-1,4) $>$ homopolycyanurates of Pr-1,2 (HPPr-1,2). The thermal stability of the polycyanurates increased as follows: HPTEG $<\mathrm{HPPr}-1,2<\mathrm{HPDEG} \approx \mathrm{HPBu}-$ 1,4 < HPEG $<$ HPDHA-1,8 < HPDHA-1,4 < HPDHN-1,7. Polymer Journal (2012) 44, 401-409; doi:10.1038/pj.2011.146; published online 14 March 2012

Keywords: carbazole; density; polyamides; solubility; thermogravimetric analysis (TGA)

\section{INTRODUCTION}

The use of polymers is rapidly increasing in nearly every region of the world. Research in the area of $s$-triazine derivatives, which has led to fundamental knowledge of novel polymers, has become increasingly widespread in recent years. Current research and development in this field involves studying the ways in which polymers can be modified to make them suitable for specific applications. Research is now focused on materials that are not only stable but also can be easily processed, and therefore, have a practical use.

A typical approach to research in this field is to utilize a stable ring system, such as triazine, and to search for a method to connect this ring-system nucleus with a thermally stable linkage, such as an ether linkage or an aliphatic linkage, to impart flexibility and solubility along with thermal stability. A review of the literature reveals that $s$-triazine and its derivatives have been known since 1873 , but their incorporation into polymer chains was not extensively studied and utilized until the late 1950s. Nakamura et al. ${ }^{1}$ synthesized various polycyanurates by interfacial polycondensation and studied the relationship between their chemical structure and their thermal and mechanical properties. Polymers containing an $s$-triazine ring in their main chain have certain properties, including high resistance to heat and chemical attack, good mechanical and dielectric properties and an ability to be easily processed..$^{2}$ In addition, they are generally infusible and insoluble. Various polycyanurates are used in structural composites with glass or carbon fibers, in casting resins and binders for miscellaneous organic and inorganic substrates, as stabilizers for plastics or as films, fibers or lacquer components. $^{3,4}$

Recently, various polyesters and polyamides based on $s$-triazine derivatives have been synthesized in our laboratory. ${ }^{5-8}$ We have used 2-carbazol-4,6-dichloro-s-triazine (CDCT) as a monomer to explore the experimental conditions for the synthesis of polymers by an interfacial technique. The present investigation should aid in determining suitable reaction conditions, studying the effects of certain specific structural features included in the polymer backbone, investigating the kinetics of the thermal degradation of the polymers synthesized and studying the effect of the chemical structure of diols on the polymers from which they are synthesized.

\section{Materials}

The monomer CDCT was synthesized from cyanuric chloride per the reaction scheme shown in Figure 1. Fresh double-distilled water was used for the preparation of the solutions. The chloroform (Ranbaxy, Mohali, India) was washed three times with distilled water to remove traces of alcohol and was treated with anhydrous calcium chloride for $24 \mathrm{~h}$ before distilling. Benzene was treated with concentrated sulfuric acid in a separating funnel to remove the traces of thiophene and then thoroughly washed with water to remove the acid. It was then treated with anhydrous calcium chloride for $24 \mathrm{~h}$ and finally distilled. The other solvents, such as nitrobenzene, carbon tetrachloride, petroleum ether, dimethylformamide, acetone and methanol, were laboratory reagents and used as received. Chemicals including sodium hydroxide 
<smiles>CC(=O)Nc1ccc2c(c1)[nH]c1ccccc12</smiles>

Figure 1 Synthesis of CDCT.

pellets and sodium bicarbonate were used as received. Cyanuric chloride (Fluka, Mumbai, India) was purified by crystallization from pure benzene. Ethylene glycol (EG), diethylene glycol (DEG), triethylene glycol (TEG), 1,2-propanediol (Pr-1,2) and 1,4-butanediol (Bu1,4) (Merck, Mumbai, India) were used as received.

\section{EXPERIMENTAL PROCEDURE}

The polycondensation of CDCT with 1,7-dihydroxynaphthalene (DHN-1,7) has been used as a model reaction for the formation of linear aromatic polycyanurate via stirred interfacial polymerization. The formation of polycyanurate is based on the Schotten-Baumann reaction in which an acid chloride reacts with a compound containing a hydrogen atom $(-\mathrm{OH},-\mathrm{NH}$ and $-\mathrm{SH})$.

\section{Synthesis of the monomer}

A solution of cyanuric chloride $(3.69 \mathrm{gm}, 0.02 \mathrm{~mol})$ in acetone $(30 \mathrm{ml})$ was added to a three-necked flask equipped with a mechanical stirrer that contained a cooled solution of sodium bicarbonate $(2.12 \mathrm{gm})$ in distilled water $(80 \mathrm{ml})$. This procedure resulted in the formation of a cyanuric chloride slurry. A solution of carbazol $(3.34 \mathrm{gm}, 0.02 \mathrm{~mol})$ in acetone $(30 \mathrm{ml})$ was slowly added to the cold slurry of cyanuric chloride. The mixture was stirred for $2 \mathrm{~h}$ at $0-5{ }^{\circ} \mathrm{C}$ (maintained at $\mathrm{pH}$ 7.0). The white product was filtered and then washed with cold water, cold dilute hydrochloric acid and finally cold water. The yield was $80 \%$ and was reduced to $75.8 \%$ after recrystallization from chloroform. The melting point was found to be $268^{\circ} \mathrm{C}$.

This reaction did not go to completion when carried out in water, unless the cyanuric chloride used was in a finely divided state. The yield of the polymer can be improved substantially if the cyanuric chloride is freshly precipitated by pouring its acetone or dioxane solution into ice water. The use of an aqueous system allows the product to be easily isolated in higher yields. ${ }^{9}$

\section{Synthesis of polycyanurates}

In the present investigation, all of the polycyanurates have been synthesized by an interfacial polycondensation technique with stirring.

Synthesis of homopolycyanurates of DHN-1,7. A mixture of DHN-1,7 ( $1.60 \mathrm{gm}, 0.01 \mathrm{~mol})$, sodium hydroxide $(0.8 \mathrm{gm}, 0.02 \mathrm{~mol})$ and benzyl dimethyl hexadecylammonium chloride in $60 \mathrm{ml}$ of double-distilled water was stirred vigorously at $25 \pm 2{ }^{\circ} \mathrm{C}$. A solution of CDCT $(3.18 \mathrm{gm}, 0.01 \mathrm{~mol})$ in $30 \mathrm{ml}$ of chloroform was rapidly added to the aqueous solution and the emulsion was stirred vigorously for $8 \mathrm{~h}$ at $25 \pm 2{ }^{\circ} \mathrm{C}$. The contents were transferred to a separating funnel and the aqueous layer was removed. The chloroform layer was washed with water and was combined with an excess of methanol from which the polymer precipitated out. The polymer was filtered and washed thoroughly with water and methanol. Approximately $76 \%$ of the theoretical yield of the polymer was obtained.

The polycyanurates of 1,4-dihydroxyanthraquinone (DHA-1,4), EG, DEG, TEG, Pr-1,2, Bu-1,4 and DHA-1,8 were synthesized under the reaction conditions optimized for the homopolymer of DHN-1,7, as shown in Figure 2.

Synthesis of homopolycyanurates of EG. A mixture of CDCT (3.18 g, $0.01 \mathrm{~mol}$ ) was heated to approximately $150^{\circ} \mathrm{C}$ in a minimal quantity of dimethylformamide $(10 \mathrm{ml})$. Cetrimide $(0.25 \mathrm{~g})$ was added, followed by EG $(0.62 \mathrm{~g}, 0.01 \mathrm{~mol})$. The temperature was raised to $165^{\circ} \mathrm{C}$ and maintained at that level for $8-10 \mathrm{~h}$. The reaction mixture was cooled and poured with constant stirring into ice<smiles>O=[N+]([O-])O[Na]</smiles><smiles></smiles>

Figure 2 Synthesis of homopolycyanurates of DHN-1,7.

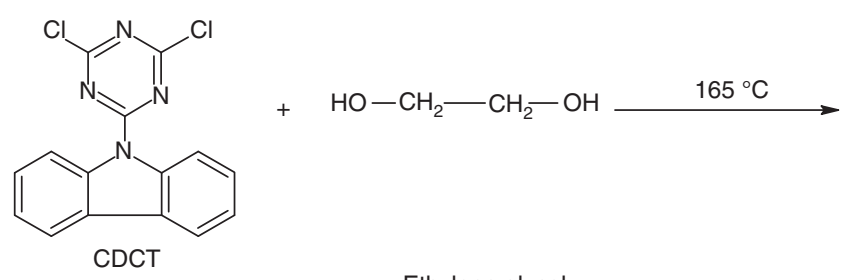<smiles>CC(C)(C)OCCOc1nc(OC(C)(C)C)nc(-n2c3ccccc3c3ccccc32)n1</smiles>

Figure 3 Synthesis of homopolycyanurates of EG.

water. The solid was filtered, washed with hot water and then methanol, and then dried. The yield was approximately $74 \%$.

The polycyanurates of EG, DEG, TEG, Pr-1,2, Bu-1,4 were synthesized under the reaction conditions optimized for the homopolymer of DHN-1,7, as shown in Figure 3.

\section{Methods of study}

The densities of the polymers were determined pycnometrically at $25^{\circ} \mathrm{C}$ by the use of a suspension method, which is well suited to powdered samples. ${ }^{10}$

The infrared (IR) spectra of each polymer was recorded using a KBr disk on a Perkin-Elmer Spectrophotometer 781 (Perkin-Elmer, Thane, India). For this, AR-grade $\mathrm{KBr}$ was fused at red heat and was thoroughly ground. A 250-mg sample of $\mathrm{KBr}$ and $1.0 \mathrm{mg}$ of the polymer were thoroughly mixed and ground in a mortar with glass beads. The ground mixture was then transferred to a mold and a disk was prepared by pressing it in a hydraulic press. ${ }^{11}$ The disks prepared in this manner were used to obtain the IR spectra of the samples.

The viscosities of the dilute polymer solutions were conveniently measured using a Ubbelohde-type capillary viscometer. ${ }^{12}$ Nuclear magnetic resonance (NMR) spectra were recorded on a Perkin-Elmer Model-32 ${ }^{1} \mathrm{H}$-NMR Spectrometer $(300 \mathrm{MHz})$ to determine the structure of the polymers. 
Thermogravimetric analysis of the polymers was carried out on DuPont TA 9900 computerized thermal analyzer (Mumbai, India). This system is comprised of the TC 10A TA processor, a control unit for the thermal analysis system TA 9900 and a TG-50 thermo balance. The TG-50 thermo balance is a microbalance (reading up to $1 \mu \mathrm{g}$ ) fixed on a furnace controlled by the TA processor. A covered alumina crucible was used as the sample carrier. A dried polymer sample was powdered to increase its surface area. The powder was loosely poured into the alumina crucible and was dispersed to counteract the poor thermal conductivity. All of the polymers were thermogravimetrically analyzed at a heating rate of $10^{\circ} \mathrm{Cmin}^{-1}$. For each of these experiments, small samples (5$10 \mathrm{mg}$ ) were used to minimize the thermal gradient within the sample.

\section{RESULTS AND DISCUSSION}

In this work, the physical properties of the polycyanurates were investigated, including their yield, color, solubility, density, viscosity, temperature characteristics, IR spectral characteristics, NMR shifts and the activation energy of the thermal decomposition.

\section{Yield and color}

The yields of the polycyanurates varied from 56 to $78 \%$ depending on the reactivity of the diol component in the polymer chain. The highest yield of $78 \%$ was obtained for homopolycyanurates of DHA-1,8 (HPDHA-1,8) and the lowest yield of 56\% for homopolycyanurates of EG (HPEG).

Table 1 Yield, color and density of copolyamides

\begin{tabular}{lclc}
\hline Polymer & Yield (\%) & Color & Density $\left(\mathrm{gm} \mathrm{cm}^{-3}\right)$ \\
\hline HPDHN-1,7 & 76 & White powdery & 1.233 \\
HPDHA-1,4 & 72 & Reddish brown & 1.244 \\
HPEG & 56 & Light brown & 1.186 \\
HPDEG & 59 & Light brown & 1.209 \\
HPTEG & 61 & Light brown & 1.221 \\
HPPr-1,2 & 58 & Light brown & 1.195 \\
HPBu-1,4 & 62 & Light brown & 1.199 \\
HPDHA-1,8 & 78 & Reddish brown & 1.247 \\
\hline
\end{tabular}

Abbreviations: HPBu-1,4, homopolycyanurates of 1,4-butanediol; HPDEG, homopolycyanurates of diethylene glycol; HPDHA-1,4, homopolycyanurates of 1,4-dihydroxyanthraquinone; HPDHA1,8 , homopolycyanurates of 1,8 -dihydroxyanthraquinone; $\mathrm{HPDHN}-1,7$, homopolycyanurates of 1,7-dihydroxynaphthalene; HPEG, homopolycyanurates of ethylene glycol; HPPr-1,2, homopolycyanurates of 1,2-propanediol; HPTEG, homopolycyanurates of triethylene glycol.
The colors of the polycyanurates synthesized were influenced by the color and stereochemistry of the diols used.

The yield and color of all the polycyanurates are summarized in Table 1.

\section{Density}

The density of each of the eight polycyanurates was determined using a pycnometer at $25 \pm 3{ }^{\circ} \mathrm{C}$ by suspending the polycyanurate in a liquid mixture of carbon tetrachloride and petroleum ether. This liquid system was found to be inert with respect to the polycyanurates because they remained in a state of suspension for a prolonged time. The estimated accuracy of the density measurements is $\pm 0.001 \mathrm{~g} \mathrm{~cm}^{-1}$. The density of the polycyanurates varied from 1.186 to $1.247 \mathrm{~g} \mathrm{~cm}^{-3}$. The highest density was displayed by HPDHA-1,8, whereas the lowest was displayed by HPEG. The densities of the polycyanurates varied with the differing chemical properties of the diols used.

The sequence of the density of the polycyanurates is as follows:

HPEG $<$ homopolycyanurates of Pr-1,2 (HPPr-1,2) < homopolycyanurates of Bu-1,4 (HPBu-1,4)<homopolycyanurates of DEG (HPDEG) < homopolycyanurates of TEG (HPTEG) < homopolycyanurates of DHN-1,7 (HPDHN-1,7) $<$ homopolycyanurates of DHA-1,4 (HPDHA-1,4) < HPDHA-1,8.

The density of each polycyanurate is summarized in Table 1 .

\section{Solubility}

Solubility tests were conducted using the experimental technique described as follows:

A 30-50-mg sample of each polymer was placed into a small test tube and $1 \mathrm{ml}$ of solvent was added. The mixtures were stored at $25^{\circ} \mathrm{C}$ with occasional shaking. The formation of streaks during shaking indicated dissolution. The polymer samples that swelled without dissolving at $25{ }^{\circ} \mathrm{C}$ were heated to $50{ }^{\circ} \mathrm{C}$ to affect the process of dissolution. Several solvents were tested for their ability to dissolve the polymer, as solubility can provide a good deal of information about the polymer. ${ }^{13-15}$ The data on the relative solubility of polycyanurates are shown in Table 2 and information is presented for aliphatic chlorinated solvents such as chloroform, methylene chloride, dichloroethane, trichloroethane, tetrachloroethane, dimethylformamide and nitrobenzene. Acetone was also found to be an effective solvent for

Table 2 Solubility of polycyanurates in various solvents

\begin{tabular}{|c|c|c|c|c|c|c|c|c|}
\hline Solvent & HPDHN-1,7 & HPDHA-1,4 & HPEG & HPDEG & HPTEG & HPPr-1,2 & HPBu-1,4 & HPDHA-1,8 \\
\hline Chloroform & \pm+ & \pm+ & ++ & ++ & ++ & ++ & ++ & \pm+ \\
\hline Dichloroethane & \pm+ & \pm+ & ++ & ++ & ++ & ++ & ++ & \pm+ \\
\hline Dichloromethane & \pm+ & \pm+ & ++ & ++ & ++ & ++ & ++ & \pm+ \\
\hline Trichloroethylene & \pm+ & \pm+ & ++ & ++ & ++ & ++ & ++ & \pm+ \\
\hline Tetrachloroethane & \pm+ & \pm+ & \pm+ & \pm+ & \pm+ & ++ & ++ & \pm+ \\
\hline Dimethylformamide & ++ & ++ & ++ & ++ & ++ & ++ & ++ & ++ \\
\hline Dimethyl sulfoxide & - \pm & - \pm & - \pm & - \pm & - \pm & - \pm & - \pm & - \pm \\
\hline Dioxane & ++ & ++ & ++ & ++ & ++ & ++ & ++ & ++ \\
\hline Nitrobenzene & ++ & ++ & ++ & ++ & ++ & ++ & ++ & ++ \\
\hline Chlorobenzene & ++ & \pm+ & \pm+ & \pm+ & \pm+ & \pm+ & \pm+ & \pm+ \\
\hline Cyclohexanone & \pm+ & \pm+ & ++ & ++ & ++ & \pm+ & \pm+ & - \pm \\
\hline Tetrachloroethane + Phenol (50:50\%) & -- & - \pm & \pm \pm & \pm \pm & \pm \pm & - \pm & - \pm & - \pm \\
\hline
\end{tabular}

Abbreviations: HPBu-1,4, homopolycyanurates of 1,4-butanediol; HPDEG, homopolycyanurates of diethylene glycol; HPDHA-1,4, homopolycyanurates of 1,4-dihydroxyanthraquinone; HPDHA-1,8, homopolycyanurates of 1,8-dihydroxyanthraquinone; HPDHN-1,7, homopolycyanurates of 1,7-dihydroxynaphthalene; HPEG, homopolycyanurates of ethylene glycol; HPPr-1,2, homopolycyanurates of 1,2-propanediol; HPTEG, homopolycyanurates of triethylene glycol.

The first and second symbol indicates the solubility of polymer at room temperature and $50{ }^{\circ} \mathrm{C}$, respectively.

+ , soluble; - , insoluble; \pm , partly soluble. 
polycyanurates. Dimethyl sulfoxide was determined to be a very poor solvent for each of these polymers. A noteworthy point in this examination is that chlorinated solvents, having a solubility parameter in the range of $9.3-10.4 \mathrm{cal} \mathrm{cm}^{-3}$ ), are good solvents for each of the polymers. Among these chlorinated solvents, chloroform was found to be the best solvent. The high solubility of these polymers in chloroform is attributed to the hydrogen-bond donor characteristics of chloroform. Because the hydrogen atom in chloroform is highly acidic, it can participate in hydrogen-bonding interactions with the oxygen of the ether linkage. The polymers were insoluble in aliphatic and aromatic hydrocarbons, alcohols and aliphatic esters. The solubility of the polymers was observed to increase with increasing temperature. The polymers that were partly soluble at $25 \pm 3^{\circ} \mathrm{C}$ were completely dissolved at higher temperatures.

\section{IR spectroscopy}

The IR spectra of the polycyanurates exhibited several common characteristic absorption frequencies in the region of $810 \mathrm{~cm}^{-1}$ that are attributed to out-of-plane and in-plane vibrations of the $s$-triazine ring. The polycyanurates involving aromatic diols in their backbone exhibited several common characteristic bands. The bands in the region of $780-650 \mathrm{~cm}^{-1}$ may be due to out-of-plane bending vibra-

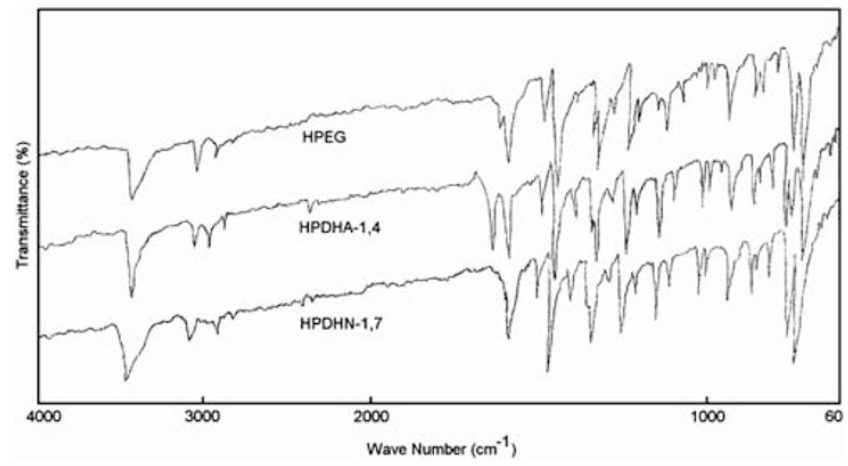

Figure 4 IR spectra of HPEG, HPDHA-1,4 and HPDHN-1,7. tions of the $\mathrm{C}-\mathrm{H}$ bonds of the aromatic rings. The bands in the 1140$980 \mathrm{~cm}^{-1}$ region may be attributed to the vibration of the aryl-ether linkage and the in-plane bending vibration of the aromatic $\mathrm{C}-\mathrm{H}$ in the polymers. The polycyanurates with aliphatic diols in their backbone exhibited bands in the region of $1125-970 \mathrm{~cm}^{-1}$ along with the above frequencies that are assigned to the aryl-ether linkages in the polycyanurates. The frequencies in the $1260-1190 \mathrm{~cm}^{-1}$ region may be attributed to vibrations involving the aryl-ether linkages. ${ }^{16-20}$ Two distinct bands, observed at approximately 1330 and $1340 \mathrm{~cm}^{-1}$, respectively, along with several bands in the $1600-1590 \mathrm{~cm}^{-1}$ region are attributed to the aromatic $\mathrm{C}-\mathrm{N}$ stretching linkage. The bands in the region of $1490-1400 \mathrm{~cm}^{-1}$ are attributed to the skeletal ring-stretching vibrations of the aromatic and heteroaromatic rings. The bands appearing in the region of $2910-2820 \mathrm{~cm}^{-1}$ are assigned as saturated aliphatic C-H stretching vibrations with an absorption band at $\sim 3060 \mathrm{~cm}^{-1}$.

It was difficult to distinguish between polycyanurates, such as HPDHN-1,7, based on frequencies in the regions of 1670-1600 and $1620-1580 \mathrm{~cm}^{-1}$, in which the ring-stretching vibrations of substituted naphthalene appear, because these regions are heavily crowded by ring-stretching vibrations of the phenyl ring. IR spectra of HPEG, HPDHA-1,4 and HPDHN-1,7 are shown in Figure 4.

The IR absorption frequencies due to various functional groups are presented in Table 3.

\section{NMR spectroscopy}

The NMR spectra of the polycyanurates derived from CDCT and various diols indicate a multiplet band at approximately 6.80$7.93 \mathrm{ppm}$ due to the presence of protons in the substituted aromatic ring system. Figure 5 shows NMR spectrum of HPDHN-1,7. The chemical shift for polycyanurate is presented in Table 4 .

\section{Viscosity}

Dilution-solution viscosity measurements were carried out using a Ubbelohde suspended-level kinematic viscometer (Cannon Instrument Co., State College, PA, USA).

Intrinsic, reduced and inherent viscosities were determined at various concentrations for each of the eight polycyanurates at

Table 3 Infrared absorption frequencies $\left(\mathrm{cm}^{-1}\right)$ of polycyanurates

\begin{tabular}{|c|c|}
\hline Polymers & Infrared absorption frequencies $\left(\mathrm{cm}^{-1}\right)$ \\
\hline HPDHN-1,7 & $\begin{array}{l}\text { 650(w), 720(s), 780(s), 810(s), 860(s), 1000(m), 1010(s), 1110(s), 1210(s), 1230(s), 1330(s), 1600(s), 1400(m), 1450(s), 1490(s), } \\
2820(w), 2910(s), 3020(w), 3440(v b)\end{array}$ \\
\hline HPDHA-1,4 & $\begin{array}{l}\text { 650(w), 720(s), 760(s), 780(s), 810(s), 860(s), 980(s), 1020(m), 1080(s), 1210(s), 1230(s), 1330(s), 1590(s), 1400(m), 1450(s), } \\
1490(\mathrm{~s}), 1630(\mathrm{~s}), 2820(\mathrm{w}), 2910(\mathrm{~s}), 3040(\mathrm{w}), 3100(\mathrm{w}), 3420(\mathrm{vb})\end{array}$ \\
\hline HPEG & $\begin{array}{l}\text { 650(w), 720(s), 780(s), 810(s), 835(s), 860(s), 970(s), 1090(m), 1120(s), 1200(w), 1240(s), 1340(s), 1600(s), 1400(m), 1450(s), 1490(s), } \\
2820(w), 2910(s), 3060(w), 3440(v b)\end{array}$ \\
\hline HPDEG & $\begin{array}{l}\text { 650(w), 720(s), 780(s), 810(s), 835(s), 860(s), 980(s), 1090(w), 1125(s), 1200(m), 1240(s), 1340(s), 1600(s), 1400(m), 1450(s), 1490(s), } \\
2820(w), 2910(s), 3040(w), 3440(v b)\end{array}$ \\
\hline HPTEG & $\begin{array}{l}\text { 650(w), 720(s), 780(s), 810(s), 835(s), 860(s), 980(s), 1040(s), 1100(w), 1120(m), 1190(m), 1240(s), 1260(s), 1340(s), 1600(s), 1400(m), } \\
1450(\mathrm{~s}), 1490(\mathrm{~s}), 2820(\mathrm{w}), 2910(\mathrm{~s}), 3060(\mathrm{w}), 3440(\mathrm{vb})\end{array}$ \\
\hline HPPr-1,2 & $\begin{array}{l}\text { 650(w), 720(s), 780(s), 810(s), 835(s), 860(s), 980(s), 1040(s), 1100(w), 1120(m), 1210(m), 1250(s), 1340(s), 1600(s), 1400(m), 1450(s), } \\
1490(\mathrm{~s}), 2820(\mathrm{w}), 2910(\mathrm{~s}), 3060(\mathrm{w}), 3440(\mathrm{vb})\end{array}$ \\
\hline HPBu-1,4 & $\begin{array}{l}\text { 650(w), 720(s), 780(s), 810(s), 835(s), 860(s), 980(s), 1040(s), 1100(w), 1120(m), 1205(m), 1240(s), 1340(s), 1600(s), 1400(m), 1450(s), } \\
1490(\mathrm{~s}), 2820(\mathrm{w}), 2910(\mathrm{~s}), 3060(\mathrm{w}), 3440(\mathrm{vb})\end{array}$ \\
\hline HPDHA-1,8 & $\begin{array}{l}\text { 650(w), 720(s), 760(m), 780(s), 810(s), 860(s), 980(s), 1010(m), 1080(s), 1120(m), 1205(w), 1240(s), 1330(s), 1590(s), 1400(m), 1450(s), } \\
1490(\mathrm{~s}), 1630(\mathrm{~s}), 2820(\mathrm{~m}), 2910(\mathrm{~s}), 3060(\mathrm{w}), 3410(\mathrm{vb})\end{array}$ \\
\hline
\end{tabular}

Abbreviations: HPBu-1,4, homopolycyanurates of 1,4-butanediol; HPDEG, homopolycyanurates of diethylene glycol; HPDHA-1,4, homopolycyanurates of 1,4-dihydroxyanthraquinone; HPDHA-1,8, homopolycyanurates of 1,8-dihydroxyanthraquinone; HPDHN-1,7, homopolycyanurates of 1,7-dihydroxynaphthalene; HPEG, homopolycyanurates of ethylene glycol; HPPr-1,2, homopolycyanurates homopolycyanurates of 1,8-dihydroxyanthraquinone; HPDHN-1,7, hom
of 1,2-propanediol; HPTEG, homopolycyanurates of triethylene glycol. Where (s), strong; $(\mathrm{m})$, medium; (w), weak; and $(\mathrm{vb})$, very broad. 
Table 4 NMR spectral characteristics of polycyanurates

\begin{tabular}{ll}
\hline Polymer & Chemical shift $(\delta$ p.p.m. $)$ \\
\hline HPDHN-1,7 & $6.80-7.48(\mathrm{~m}, \mathrm{Ar}-\mathrm{H})$ \\
HPDHA-1,4 & $7.05-7.93(\mathrm{~m}, \mathrm{Ar}-\mathrm{H})$ \\
HPEG & $4.74\left(\mathrm{~s}, \mathrm{CH}_{2}\right), 6.93-7.64(\mathrm{~m}, \mathrm{Ar}-\mathrm{H})$ \\
HPDEG & $3.87\left(\mathrm{t}, \mathrm{CH}_{2}\right), 4.46\left(\mathrm{t}, \mathrm{CH}_{2}\right), 6.90-7.55(\mathrm{~m}, \mathrm{Ar}-\mathrm{H})$ \\
HPTEG & $3.50\left(\mathrm{~s}, \mathrm{CH}_{2}\right), 3.78\left(\mathrm{t}, \mathrm{CH}_{2}\right), 4.53\left(\mathrm{t}, \mathrm{CH}_{2}\right), 7.18-7.74(\mathrm{~m}, \mathrm{Ar}-\mathrm{H})$ \\
HPPr-1,2 & $1.31\left(\mathrm{~d}, \mathrm{CH}_{3}\right), 4.12\left(\mathrm{~d}, \mathrm{CH}_{2}\right), 5.33(\mathrm{~m}, \mathrm{CH}), 7.22-7.70(\mathrm{~m}, \mathrm{Ar}-\mathrm{H})$ \\
HPBu-1,4 & $2.18\left(\mathrm{t}, \mathrm{CH}_{2}\right), 3.76\left(\mathrm{t}, \mathrm{CH}_{2}\right), 7.12-7.63(\mathrm{~m}, \mathrm{Ar}-\mathrm{H})$ \\
HPDHA-1,8 & $6.93-7.57(\mathrm{~m}, \mathrm{Ar}-\mathrm{H})$
\end{tabular}

Abbreviations: HPBu-1,4, homopolycyanurates of 1,4-butanediol; HPDEG, homopolycyanurates of diethylene glycol; HPDHA-1,4, homopolycyanurates of 1,4-dihydroxyanthraquinone; HPDHA1,8 , homopolycyanurates of 1,8-dihydroxyanthraquinone; HPDHN-1,7, homopolycyanurates of 1,7-dihydroxynaphthalene; HPEG, homopolycyanurates of ethylene glycol; HPPr-1,2, homopolycyanurates of 1,2-propanediol; HPTEG, homopolycyanurates of triethylene glycol, NMR, nuclear magnetic resonance.

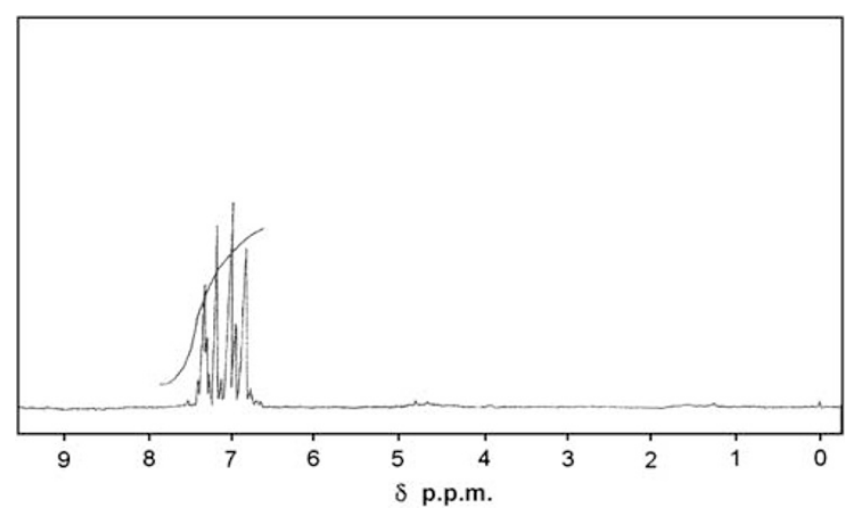

Figure 5 NMR spectrum of HPDHN-1,7.
$25 \pm 0.1^{\circ} \mathrm{C}$. The reduced and inherent viscosities were calculated from experimental data. Typical Huggins and Kraemer plots were used to obtain the intrinsic viscosity for each of the polycyanurates. The solution viscosities of HPDHN-1,7 at different concentrations are shown in Table 5.

Intrinsic viscosity, reduced viscosity and inherent viscosity, along with Huggin's and Kraemer's constants for a $1 \%$ solution for each of the polycyanurates are shown in Table 6.

Examination of the intrinsic viscosities of the polycyanurates reveals that HPDHN-1,7 has the highest solution viscosity, and thus the highest molecular weight among all of the polycyanurates, whereas the HPPr-1,2 has the lowest solution viscosity, as shown in Figure 6. The intrinsic viscosity of the polycyanurates follows the sequence given below:

HPDHN-1,7 $>$ HPDHA-1,8 $>$ HPDHA-1, $4>$ HPEG $>$ HPEG $>$ HP-

DEG $>$ HPTEG $>$ HPBu-1,4 $>$ HPPr- 1,2 .

This order may be attributed to the basicity strength of the diols. Thus, the reactivity towards a nucleophilic substitution reaction is higher for a less acidic diol. DHN-1,7 is less acidic, therefore, it is the most reactive among the diols used in the present investigation. In contrast, Pr-1,2 is highly acidic, thus, it is a less reactive diol. The reduced viscosity values may also be reflected in the order of reactivity with respect to the nucleophilic substitution reactions of these diols.

\section{Thermal decomposition characteristics}

Thermal stability is the most desirable property of a polymer. If the thermal stability of a polymer is increased, it is accompanied by an increase in its strength modules, rigidity and softening temperature. The thermal degradation reaction of a specific polymer depends upon a large number of variables, including the decomposition temperature, heating rate, rate of removal of volatile products from the reaction

Table 5 Solution viscosity of polycyanurate of DHN-1,7 (HPDHN-1,7)

Solvent: chloroform

Temperature: $25^{\circ} \mathrm{C}$

$t_{0}=91 \mathrm{~s}$

\begin{tabular}{|c|c|c|c|c|c|}
\hline Conc. (C) $g d l^{-1}$ & Flow time $t$ sec & $\eta_{r e}=t / t_{o}$ & $\eta_{s p}=\eta_{r e l}-1$ & Reduced viscosity $\left(\eta_{s p} / C\right)$ & In $\eta_{r e} / C$ \\
\hline 0.2 & 100.6 & 1.1055 & 0.1055 & 0.5275 & 0.5015 \\
\hline 0.4 & 110.8 & 1.2176 & 0.2176 & 0.5440 & 0.4945 \\
\hline 0.6 & 121.5 & 1.3352 & 0.3352 & 0.5586 & 0.4922 \\
\hline 0.8 & 132.5 & 1.4560 & 0.4560 & 0.5700 & 0.4696 \\
\hline 1.0 & 144.5 & 1.5879 & 0.5879 & 0.5879 & 0.4624 \\
\hline
\end{tabular}

Table 6 Various viscosity values and constants of Huggins and Kraemer equation for polycyanurates

\begin{tabular}{|c|c|c|c|c|c|}
\hline Polymer & Intrinsic viscosity $\eta$ & Reduced viscosity $\eta_{s p} / C\left(d / g^{-1}\right)$ & Inherent viscosity (In $\left.\eta_{r e} / C\right)$ & Huggins constant $(\mathrm{K})$ & Kraemer constant $(\beta)$ \\
\hline HPDHN-1,7 & 0.5125 & 0.5879 & 0.4624 & 0.2871 & 0.1905 \\
\hline HPDHA-1,4 & 0.4450 & 0.5020 & 0.4058 & 0.2879 & 0.1980 \\
\hline HPEG & 0.4215 & 0.4747 & 0.3850 & 0.2994 & 0.2050 \\
\hline HPDEG & 0.3795 & 0.4238 & 0.3542 & 0.3076 & 0.1756 \\
\hline HPTEG & 0.3550 & 0.3850 & 0.3220 & 0.2381 & 0.2619 \\
\hline HPPr-1,2 & 0.2800 & 0.3000 & 0.2610 & 0.2551 & 0.2420 \\
\hline HPBu-1,4 & 0.3180 & 0.3480 & 0.2980 & 0.2967 & 0.1978 \\
\hline HPDHA-1,8 & 0.4655 & 0.5181 & 0.4100 & 0.2423 & 0.2561 \\
\hline
\end{tabular}

Abbreviations: HPBu-1,4, homopolycyanurates of 1,4-butanediol; HPDEG, homopolycyanurates of diethylene glycol; HPDHA-1,4, homopolycyanurates of 1,4-dihydroxyanthraquinone; HPDHA-1,8, Abbreviations: HPBu-1,4, homopolycyanurates of 1,4-butanediol; HPDEG, homopolycyanurates of diethylene glycol; HPDHA-1,4, homopolycyanurates of 1,4-dihydroxyanthraquinone; HPDHA-1,8, of 1,2-propanediol; HPTEG, homopolycyanurates of triethylene glycol.

Concentration of polymer solution is $1.0 \mathrm{~g} \mathrm{dl}^{-1}$ 


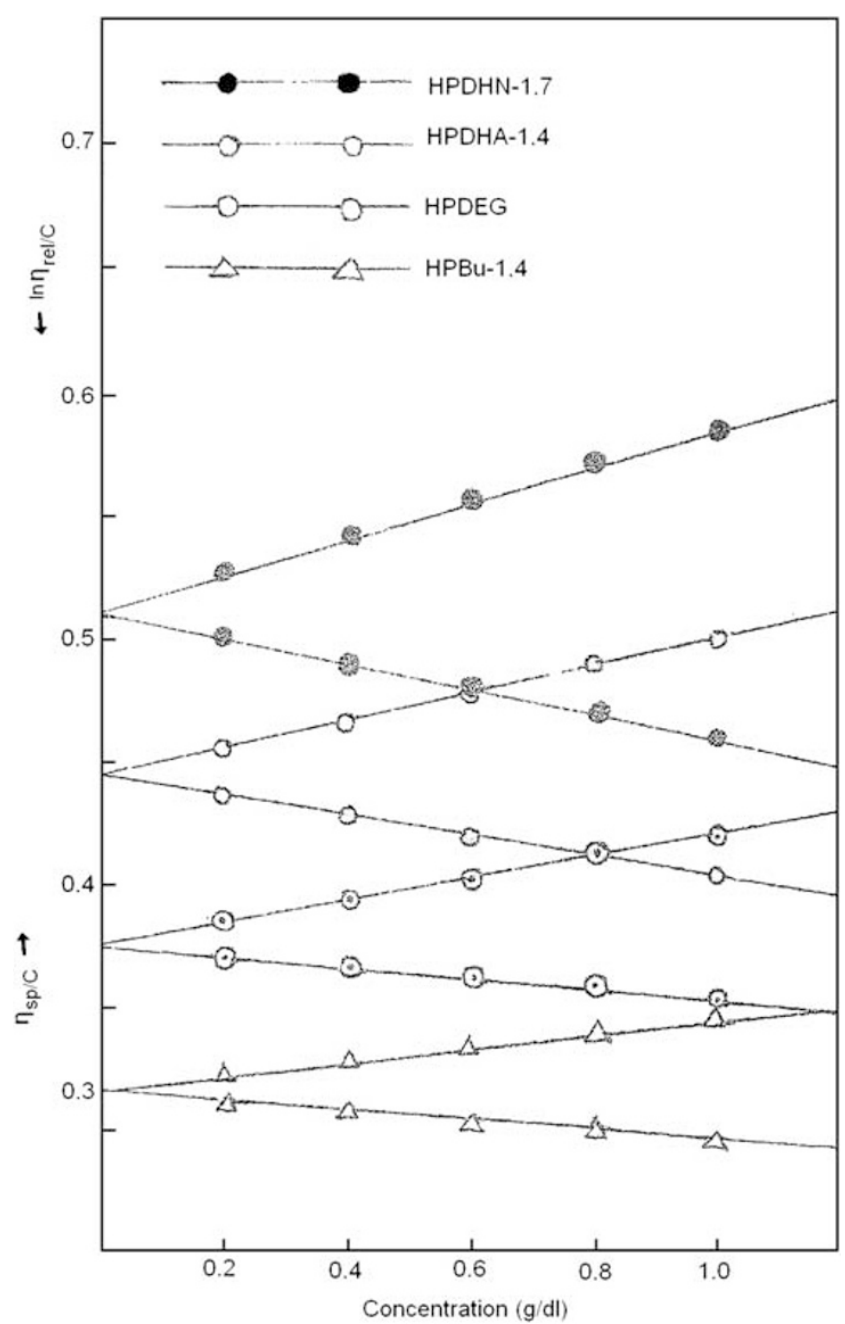

Figure 6 Huggins and Kraemer plots of intrinsic viscosity.

zone, softening and melting points of the polymer, sample size and dimension, presence of oxygen in the atmosphere or absorbed into the sample, other occluded impurities, the initiator used and the mechanism of termination in the original preparation of the polymer. Therefore, the thermal decomposition is performed under an inert atmosphere with carefully purified polymers in the form of finely divided powders.

Figure 7 shows thermogram for HPPr-1,2, HPDHA-1,4, HPDEG and HPDHN-1,7, whereas Figure 8 shows thermogram for HPDHA1,8, HPTEG, HPEG and HPBu-1,4.

Thermogravimetric curves were obtained at a scan rate of $10{ }^{\circ} \mathrm{C} \mathrm{min}^{-1}$ for each of the polycyanurates.

HPDHN-1,7 begins to decompose at approximately $230^{\circ} \mathrm{C}$. A slight initial weight loss below $230^{\circ} \mathrm{C}$ may be due to absorbed moisture or an associated solvent. The polymer decomposes with a rapid weight loss $\left(47.50 \%\right.$ of its weight) in the temperature range of $360-500^{\circ} \mathrm{C}$. The maximum rate of weight loss occurs at $440{ }^{\circ} \mathrm{C}$. Above $530{ }^{\circ} \mathrm{C}$, the second decomposition step (having a slower rate of weight loss compared with the first step) commences with $31.50 \%$ loss of its weight. The overall decomposition leaves approximately $20.75 \%$ of residue remaining. HPDHA-1,4 exhibits two distinct stages of decomposition. The first stage occurs in the range of $310-500{ }^{\circ} \mathrm{C}$. The maximum rate of decomposition occurs at $400{ }^{\circ} \mathrm{C}$. The polymer

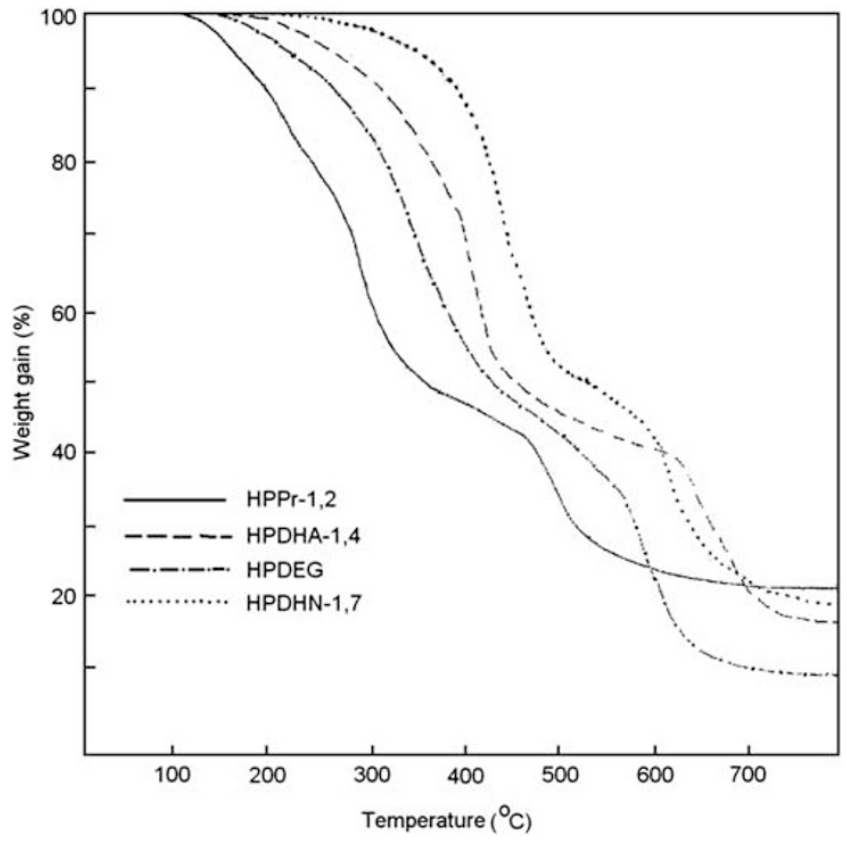

Figure 7 Thermogram for HPPr-1,2, HPDHA-1,4, HPDEG and HPDHN-1,7.

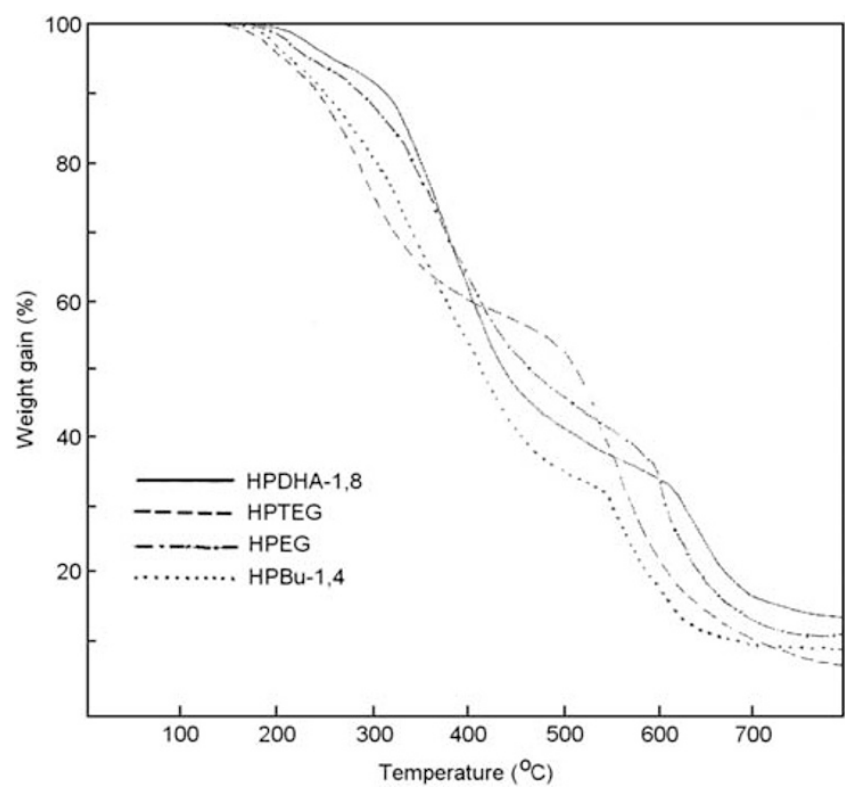

Figure 8 Thermogram for HPDHA-1,8, HPTEG, HPEG and HPBu-1,4.

loses $56.5 \%$ of its weight in this step. The second stage commences in the range of $610-700^{\circ} \mathrm{C}$. The maximum rate of decomposition occurs at $640{ }^{\circ} \mathrm{C}$. The polymer loses $26.0 \%$ of its weight in this step and the overall decomposition ultimately leaves approximately $17.5 \%$ of the residue.

The thermogram of HPEG exhibits two distinct steps. The decomposition begins at $\sim 170{ }^{\circ} \mathrm{C}$, with a maximum rate of decomposition at $370{ }^{\circ} \mathrm{C}$. The polymer loses $54.50 \%$ of its weight at up to $500^{\circ} \mathrm{C}$. The second step commences at approximately $540-700^{\circ} \mathrm{C}$, during which the polymer loses $32.75 \%$ of its weight. The maximum rate of decomposition occurs at $600{ }^{\circ} \mathrm{C}$. The overall decomposition leaves approximately $12.75 \%$ of the residue remaining. 
HPDEG loses $51.0 \%$ of its weight in the temperature range of 270 $440{ }^{\circ} \mathrm{C}$ with a maximum rate of decomposition at $340^{\circ} \mathrm{C}$. The second decomposition step commences in the range of $530-640^{\circ} \mathrm{C}$. The homopolymer loses $38.5 \%$ of its weight in this step.

The thermogram curves of HPTEG show two distinct steps of decomposition. The polymer begins to decompose at approximately $130^{\circ} \mathrm{C}$. The decomposition is marked with a rapid weight loss in the range of $210-380^{\circ} \mathrm{C}$, during which the polymer loses $38.0 \%$ of its weight. The maximum rate of decomposition occurs at $280^{\circ} \mathrm{C}$. The second decomposition step commences with a $53.5 \%$ loss of its weight, having a faster rate of weight loss compared with the first step. The overall decomposition leaves approximately $8.5 \%$ of its weight.

In the temperature range of $190-360^{\circ} \mathrm{C}, \mathrm{HPPr}-1,2$ loses $51.5 \%$ of its weight. The maximum rate of weight loss occurs at $290{ }^{\circ} \mathrm{C}$. Above $450{ }^{\circ} \mathrm{C}$, the second decomposition step commences with loss of $26.0 \%$ of its weight and has a low rate of weight loss compared with the first step. The maximum rate of weight loss occurs at $490{ }^{\circ} \mathrm{C}$. The decomposition ultimately leaves approximately $22.5 \%$ of the residue.

The overall degradation of HPBu-1,4 involves two steps. The first step encompasses a temperature range of $260-510^{\circ} \mathrm{C}$ and involves a weight loss of approximately $63.5 \%$. The rate of weight loss reaches a maximum at $340{ }^{\circ} \mathrm{C}$. The second step of the degradation commences from $520-620^{\circ} \mathrm{C}$ and involves a weight loss of approximately $25.0 \%$. The maximum rate of weight loss occurs at $560^{\circ} \mathrm{C}$.

Similarly, the overall thermal degradation of HPDHA-1,8 involves two distinct steps. At a temperature of $300^{\circ} \mathrm{C}$, the first step of degradation begins and extends up to $460{ }^{\circ} \mathrm{C}$ with a weight loss of approximately $54.0 \%$. The maximum rate of weight loss occurs at $380^{\circ} \mathrm{C}$. Above $590^{\circ} \mathrm{C}$, the second step of the decomposition commences and involves further weight loss of $31.0 \%$ at up to $700{ }^{\circ} \mathrm{C}$ with a maximum rate at $640{ }^{\circ} \mathrm{C}$.

The characteristic temperatures for the assessment of the relative thermal stability of polycyanurates, such as the initial decomposition temperature $\left(\mathrm{T}_{0}\right)$, temperature for $10 \%$ weight loss $\left(\mathrm{T}_{10}\right)$, temperature for the maximum rate of decomposition $\left(\mathrm{T}_{\max }\right)$ and temperature for half volatilization $\left(T_{s}\right)$, are represented in Table 7 .

The thermal stability of the polycyanurates studied was established on the basis of $\mathrm{T}_{\max }$ for the first step of decomposition. The thermal stability of polycyanurates increases in the following order:

HPTEG $<$ HPPr- $1,2<$ HPDEG $\approx$ HPBu- $1,4<$ HPEG $<$ HPDHA-

$1,8<$ HPDHA- $1,4<$ HPDHN-1,7.

The thermal stability based on the half-volatilization-point temperature $\left(\mathrm{T}_{\mathrm{s}}\right)$ follows a somewhat different sequence. The thermal stability increases as follows:

HPPr- $1,2<$ HPBu- $1,4<$ HPDEG $<$ HPDHA- $1,4<$ HPEG $<$ HPTE-

G $<$ HPDHA- $1,8<$ HPDHN-1,7.

The above thermal stability trends show that the polycyanurate of $\operatorname{Pr}-1,2$ (HPPr-1,2) is least stable, whereas the polycyanurate of DHN1,7 (HPDHN-1,7) possesses the highest thermal stability.

\section{Evaluation of the kinetic parameters}

Dynamic TGA thermograms obtained at a heating rate of $10{ }^{\circ} \mathrm{C} \mathrm{min}-1$ have been analyzed according to the graphical methods proposed by Broido, Horowitz-Metzger. Broido plot for thermal degradation of HPDHN-1,7 is depicted in Figure 9. Horowitz-Metzger plot for thermal degradation of HPDHN-1,7 is shown in Figure 10.

A typical application of Broido's method is exemplified for HPDHN-1,7 in Table 8, and the Horowitz-Metzger method is exemplified for HPDHN-1,7 in Table 9. The straight lines are due to linear regression analysis of the experimental data. The magnitude
Table 7 Thermal characteristics of various polycyanurates

\begin{tabular}{lcccccc}
\hline & & \multicolumn{5}{c}{$T_{\max }$} \\
\cline { 4 - 5 } Polymer & $T_{0}$ & $T_{10}$ & Step I & Step II & $T_{s}$ & Residue at $800^{\circ} \mathrm{C}$ \\
\hline HPDHN-1,7 & 230 & 380 & 440 & 610 & 525 & 20.75 \\
HPDHA-1,4 & 190 & 300 & 400 & 600 & 450 & 17.50 \\
HPEG & 170 & 280 & 370 & 600 & 475 & 12.75 \\
HPDEG & 140 & 245 & 340 & 570 & 420 & 10.50 \\
HPTEG & 130 & 240 & 280 & 560 & 510 & 08.50 \\
HPPr-1,2 & 110 & 200 & 290 & 490 & 350 & 22.50 \\
HPBu-1,4 & 150 & 250 & 340 & 560 & 415 & 11.50 \\
HPDHA-1,8 & 200 & 315 & 380 & 650 & 515 & 14.75 \\
\hline
\end{tabular}

Abbreviations: HPBu-1,4, homopolycyanurates of 1,4-butanediol; HPDEG, homopolycyanurates of diethylene glycol: HPDHA-1,4, homopolycyanurates of 1,4-dihydroxyanthraquinone, HPDHA1,8, homopolycyanurates of 1,8-dihydroxyanthraquinone; HPDHN-1,7, homopolycyan 1,7-dihydroxynaphthalene; HPEG, homopolycyanurates of ethylene glycol; HPPr-1,2, initial decomposition temperature; $T_{10}$, temperature for $10 \%$ weight loss; $T_{\max }$, maximum rate of decomposition temperature; $T_{\mathrm{s}}$, temperature for $50 \%$ weight loss.
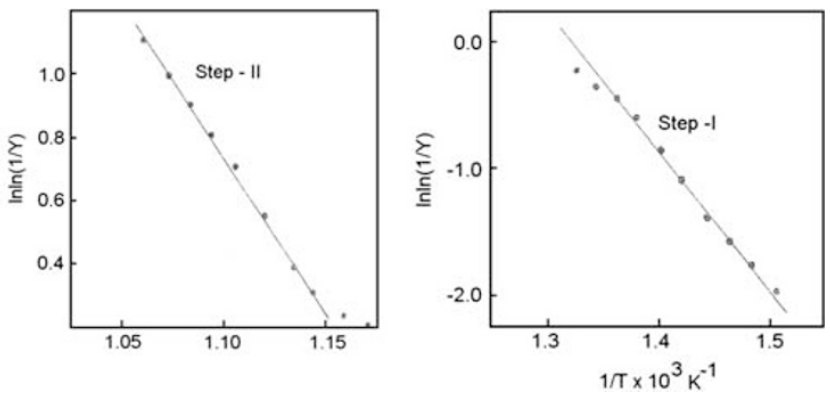

Figure 9 Broido plot for the thermal degradation of HPDHN-1,7.

of an apparent activation energy for a degradation reaction reflects the ease with which it can proceed. Therefore, activation energy may be used to approximate the relative ease of the thermal degradation of a polymer, and hence its relative thermal stability.

The thermograms of other polymers were analyzed using the same graphical method. The values of the apparent activation energy corresponding to the different degradation steps involved were evaluated from the slopes of the least-squares plots of the relevant data and are presented in Table 10. Examination of the data indicates that both the methods yield comparable values for $E$. The experimental points corresponding to the initial stage $(10 \%$ loss) of the thermal degradation tended to deviate from linearity. This deviation may be due to the fact that the decomposition of solids does not obey firstorder kinetics in the initial stages.

Of the two methods, the Broido method is expected to provide more reliable estimates of $E$ because no other temperature characteristics are involved.

The values for the activation energies for first step vary from 24.93 to $9.03 \mathrm{kcal} \mathrm{mol}^{-1}$ according to the Broido method. The values for ' $\mathrm{E}$ ' calculated according to the Horowitz-Metzger method are in good agreement with these values. The activation energies for the second step of thermal degradation in the cases of HPEG, HPDEG, HPTEG, HPPr-1,2, HPBu-1,4 and HPDHA-1,8 are higher than those of the first step, whereas in the cases of HPDHA-1,4 and HPDHN-1,7, they are lower than those of the first step. 


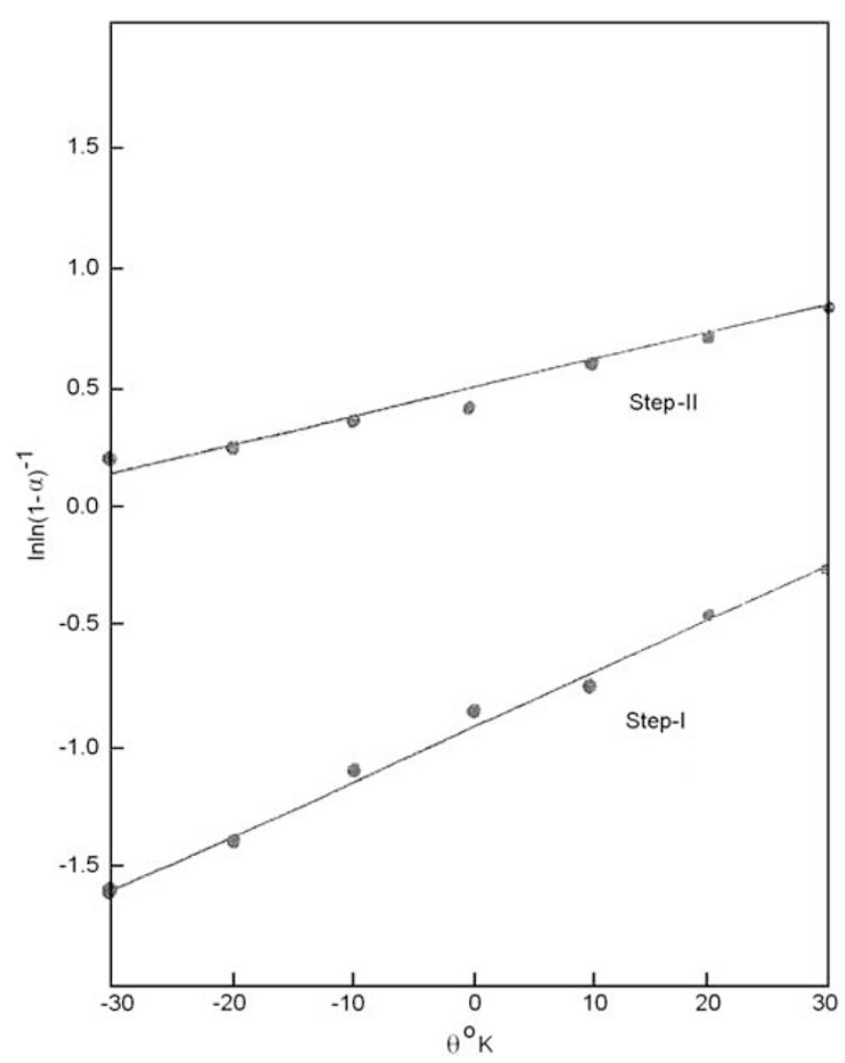

Figure 10 Horowitz-Metzger plot for the thermal degradation of HPDHN1,7 .

Table 8 Application of Broido method to thermogram of HPDHN-1,7 obtained at $10^{\circ} \mathrm{C} / \mathrm{min}$

\begin{tabular}{|c|c|c|c|c|c|c|}
\hline Temperature $\left({ }^{\circ} \mathrm{C}\right)$ & $\% W t$ & $y=\frac{W_{t}-W_{\alpha}}{W_{t}-W_{0}}$ & $1 / y$ & $\ln \ln 1 / y$ & $T^{\circ} \mathrm{K}$ & $1 / T \times 10^{3 \circ} \mathrm{K}^{-1}$ \\
\hline \multicolumn{7}{|l|}{ Step I } \\
\hline 390 & 89.50 & 0.8675 & 1.1527 & -1.9510 & & 1.5084 \\
\hline 400 & 87.50 & 0.8423 & 1.1873 & -1.7623 & & 1.4859 \\
\hline 410 & 85.50 & 0.8170 & 1.2239 & -1.5991 & & 1.4641 \\
\hline 420 & 83.00 & 0.7855 & 1.2731 & -1.4211 & & 1.4430 \\
\hline 430 & 77.00 & 0.7098 & 1.4089 & -1.0706 & & 1.4225 \\
\hline 440 & 72.50 & 0.6530 & 1.5314 & -0.8529 & & 1.4025 \\
\hline 450 & 66.00 & 0.5710 & 1.7514 & -0.5791 & & 1.3831 \\
\hline 460 & 63.00 & 0.5331 & 1.8757 & -0.4636 & & 1.3643 \\
\hline 470 & 59.00 & 0.4827 & 2.0719 & -0.3168 & & 1.3459 \\
\hline 480 & 56.50 & 0.4511 & 2.2168 & -0.2281 & & 1.3280 \\
\hline \multicolumn{7}{|l|}{ Step // } \\
\hline 580 & 44.00 & 0.2934 & 3.4086 & 0.2040 & & 1.1723 \\
\hline 590 & 43.00 & 0.2808 & 3.5618 & 0.2392 & & 1.1588 \\
\hline 600 & 41.00 & 0.2555 & 3.9136 & 0.3108 & & 1.1455 \\
\hline 610 & 39.00 & 0.2303 & 4.3425 & 0.3842 & & 1.1325 \\
\hline 620 & 34.50 & 0.1735 & 5.7636 & 0.5605 & & 1.1198 \\
\hline 630 & 31.00 & 0.1239 & 7.7317 & 0.7156 & & 1.1074 \\
\hline 640 & 29.00 & 0.1041 & 9.6061 & 0.8164 & & 1.0953 \\
\hline 650 & 27.50 & 0.0852 & 11.7407 & 0.9014 & & 1.0834 \\
\hline 660 & 26.00 & 0.0662 & 15.0952 & 0.9986 & & 1.0718 \\
\hline 670 & 24.50 & 0.0473 & 21.1333 & 1.1154 & & 1.0605 \\
\hline
\end{tabular}

Table 9 Application of Horowitz-Metzger's method to thermogram of HPDHN-1,7 obtained at $10^{\circ} \mathrm{C} \mathrm{min}-1$

\begin{tabular}{rccccr}
\hline Temperature $\left({ }^{\circ} \mathrm{C}\right)$ & $\alpha$ & $1-\alpha$ & $1 / 1-\alpha$ & $\ln \ln (1 / 1-\alpha)$ & $\theta$ \\
\hline Step I & & & & & \\
410 & 0.1830 & 0.8170 & 1.2239 & -1.5991 & -30 \\
420 & 0.2145 & 0.7855 & 1.2731 & -1.4211 & -20 \\
430 & 0.2902 & 0.7098 & 1.4089 & -1.0706 & -10 \\
440 & 0.3470 & 0.6530 & 1.5314 & -0.8529 & 0 \\
450 & 0.4290 & 0.5710 & 1.7514 & -0.5791 & 10 \\
460 & 0.4669 & 0.5331 & 1.8757 & -0.4636 & 20 \\
470 & 0.5173 & 0.4827 & 2.0719 & -0.3168 & 30 \\
& & & & & \\
Step II & & & & & -30 \\
580 & 0.7066 & 0.2934 & 3.4086 & 0.2040 & -20 \\
590 & 0.7192 & 0.2808 & 3.5618 & 0.2392 & -10 \\
600 & 0.7445 & 0.2555 & 3.9136 & 0.3108 & 10 \\
610 & 0.7697 & 0.2303 & 4.3425 & 0.3842 & 20 \\
620 & 0.8265 & 0.1735 & 5.7636 & 0.5605 & 30 \\
630 & 0.8761 & 0.1239 & 7.7317 & 0.7156 & 0.8164 \\
640 & 0.8959 & 0.1041 & 9.6061 & & \\
\hline
\end{tabular}

Table 10 Kinetic parameters for thermal decomposition of polycyanurates

\begin{tabular}{|c|c|c|c|c|}
\hline \multirow[b]{3}{*}{ Polymer } & \multicolumn{4}{|c|}{ Energy of activation $E\left(\mathrm{Kcal} \mathrm{mol}^{-1}\right)$} \\
\hline & \multicolumn{2}{|c|}{ Broido } & \multicolumn{2}{|c|}{ Horowitz-Metzger } \\
\hline & Step I & Step /I & Step I & Step II \\
\hline HPDHN-1,7 & 24.93 & 18.54 & 25.66 & 19.27 \\
\hline HPDHA-1,4 & 16.63 & 12.29 & 16.16 & 12.13 \\
\hline HPEG & 8.57 & 14.12 & 8.99 & 14.28 \\
\hline HPDEG & 10.11 & 12.66 & 10.56 & 13.03 \\
\hline HPTEG & 9.75 & 16.44 & 9.76 & 16.27 \\
\hline HPPr-1,2 & 9.03 & 10.68 & 9.30 & 11.99 \\
\hline HPBu-1,4 & 9.20 & 13.33 & 9.37 & 13.34 \\
\hline BPDHA-1,8 & 14.23 & 15.81 & 13.83 & 15.63 \\
\hline
\end{tabular}

Examination of Table 10 reveals that HPDHN-1,7 shows the highest value of activation energy for the initial stage of thermal degradation, whereas HPEG, HPPr-1,2, HPBu-1,4, HPDEG and HPTEG show lower activation energies. Taking aromaticity into account, the polycyanurates derived from naphthalene diols are expected to be the most thermally stable among all of the polymers, but only the polycyanurate involving DHN-1,7 closely follows this assumption.

Furthermore, this trend in relative stability indicates that the initial site of degradation may lie in the nature of the bridge, ' $\mathrm{X}$ ', in the aromatic diol component of the molecular chain. Thus, results based on the qualitative and semiquantitative treatment of the dynamic thermogravimetric data for the polymers indicate that inclusion of bisphenol-S and a symmetrical dihydroxynaphthalene moiety in the homopolymer backbone is advantageous for improved thermal stability. Moreover, such inclusion does not adversely affect the solubility of the resultant homopolymer. 


\section{CONCLUSIONS}

The properties of polycyanurates largely depend upon the structure and mode of their preparation. These properties are influenced by the nature of the substituents on the $s$-triazine nucleus and by the nature of the diol component of the polymer chain.

\section{ACKNOWLEDGEMENTS}

We are grateful to Atul Limited, Valsad, for providing useful chemicals.

1 Nakamura, Y., Mori, K., Tamura, K. \& Saito, Y. Relation of the chemical structure of polycyanurates to thermal and mechanical properties. J. Polym. Sci. A-1 7, 3089 (1969).

2 Tigelaar, D. M., Palker, A. E., Jackson, C. M., Anderson, K. M., Wainright, J. \& Savinell, R. F. Synthesis and properties of novel proton-conducting aromatic poly (ether sulfone)s that contain triazine groups. Macromolecules 42, 1888-1896 (2009).

3 Hamerton, I. The chemistry and Technology of Cyanate Ester Resins (Chapman and Hall: Glasgow, 1994)

4 Ismail, V. R. M. Über triazinhaltige polytetrachlorarylester. Kurzmitteilung. Die. Angew. Macromol. Chem. 21, 25-30 (1972).

5 Asundaria, S. T., Patel, P. R. \& Patel, K. C. Novel copolyesters based on s-triazine derivatives. Int. J. Polym. Mat. 58, 692-705 (2009).

6 Asundaria, S. T. \& Patel, K. C. Synthesis and characterization of novel copolyamides based on s-triazine derivatives. Int. J. Polym. Mat. 59, 370-386 (2010).

7 Asundaria, S. T., Patel, K. C. \& Patel, H. S. Synthesis and physicochemical studies of some novel homopolyesters based on s-triazine. Int. J. Polym. Mat. 59, 241-254 (2010).
8 Patel, K. C., Asundaria, S. T. \& Patel, P. R. Novel copolyamides based on s-triazine derivatives. Int. J. Polym. Mat. 59, 118-133 (2010).

9 Parsania, P. H., Shah, P. P., Patel, K. C. \& Patel, R. D. Morphology property relationships in poly (2-methoxy)cyanurate of bisphenol-C (PMCBC)/polystyrene (PS) blendsdynamicmodulus. Die Angew Macromol Chem. 138, 139-149 (1986).

10 Ashraf, S. M., Sharif, A. \& Riaz, U. A laboratory manual of polymers. vol. 1114 (I. K. International: New Delhi, 2009).

11 Silverstein, R. M. \& Webster, F. Spectrometric Identification of Organic Compounds 6th edn (Wiley: New York, 2005).

12 Ubbelohde, L. Ind. Eng. Chem. Anal. Edn 9, 85 (1935).

13 Bozdogan, A. E. A method for determination of thermodynamics and solubility parameters of polymers in dilute solutions from critical volume fractions. Polymer 44, 6427-6430 (2003).

14 Rindfleisch, F., DiNoia, T. P. \& McHugh, M. A. Solubility of polymers and copolymers in supercritical $\mathrm{CO}_{2}$. J. Phys. Chem. 100, 15581-15587 (1996).

15 Jang, B. N., Wang, D. \& Wilkie, C. A. Relationship between the solubility parameter of polymers and the clay dispersion in polymer/clay nanocomposites and the role of the surfactant. Macromolecules 38, 6533-6543 (2005).

16 Choudhary, R. B., Anand, O. N. \& Tyagi, O. S. Surface reactivity and layer analysis of chemisorbed reaction films in the surface-chemical environment of alkyl octadecenoates. J. Chem. Sci. 121, 353-360 (2009).

17 Patel, H. S. \& Patel, K. C. Copolycyanurates from 2-(N-ethyl anilino)-4,6-bis (phenoxy2-carbonyl chloride)-s-triazine: synthesis and characterization. Iran. Polym. J. 15, 505-513 (2006).

18 Zulfiqar, S., Ahmad, Z. \& Ilyas Sarwar, M. Soluble aromatic polyamide bearing ether linkages: synthesis and characterization. Coll. Polym. Sci. 285, 1749-1754 (2007)

19 Yue, X., Zhang, H., Chen, W., Wang, Y., Zhang, S., Wang, G. \& Jiang, G. Crosslinkable fully aromatic poly(aryl ether ketone)s bearing macrocycle of aryl ether ketone. Polymer 48, 4715-4722 (2007).

20 Wang, C. S. \& Leu, T. S. Novel bismaleimide with naphthalene and aryl ether linkages. J. Appl. Poly. Sci. 73, 833-839 (1999). 